\title{
Machine Learning Algorithms and Wireless Sensor Network Applied to Medical Diagnosis: A Systematic Review
}

\author{
Ibrahim Goni \\ Department of Computer Science, Faculty of Science, Adamawa State University, Mubi, Nigeria \\ Email address: \\ algonis1414@gmail.com \\ To cite this article: \\ Ibrahim Goni. Machine Learning Algorithms and Wireless Sensor Network Applied to Medical Diagnosis: A Systematic Review. American \\ Journal of Electromagnetics and Applications. Vol. 7, No. 2, 2019, pp. 25-33. doi: 10.11648/j.ajea.20190702.13
}

Received: September 18, 2019; Accepted: September 27, 2019; Published: December 17, 2019

\begin{abstract}
In this research work systematic approach were used to conduct a survey on recent contributions of the authors that applied Machine learning algorithms or computational intelligence, Artificial intelligence and soft computing techniques such as Artificial Neural Network, Fuzzy logic Genetic algorithm, Artificial Immune System Swarm intelligence among others or any combination of these techniques Neuro-fuzzy, Adaptive Neuro-fuzzy, Neuro-genetic, fuzzy-genetic, and so on that is soft computing to medical diagnosis and also a systematic review on the application of wireless sensor network, wireless sensor is a veritable embedded system with a wireless communication function, and that is capable to: Collect physical quantities such as heat, humidity, temperature, vibration, radiation, sound, light, movement, etc. Convert them into digital values which are sent as sensed data to a remote processing station or base station (WSN) to medical and health care delivery. However the survey believed that combining wireless sensor network with soft computing techniques, artificial intelligence techniques can perform well in providing health care services compare to one technique because any of these techniques has certain limitations but together perhaps they two or three techniques connected together would reduce error to a minimum level.
\end{abstract}

Keywords: Computational Intelligence, Artificial Intelligence, Soft Computing, Wireless Sensor Network

\section{Introduction}

Machine Learning algorithms are also known as Computational Intelligence such as Artificial Neural Network (ANN), Fuzzy logic (FL), Genetic algorithm (GA), Swarm Intelligence (SI), Artificial Immune System (AIS), Simulated Annealing (SA), Genetic Programming (GP), Evolutionary Strategy (ES), Evolutionary Programming (EP), Multi Agent System (MAS) Ant Colony Optimization (ACO) and Particle Swarm Optimization (PSO) Probabilistic Reasoning (PB) and the combination of two or three of these techniques resulted in hybrid system technically known as soft computing which includes Neuro-fuzzy, is a combination of Neural network and fuzzy logic, Neuro-Genetic which is a combination of Neural Network and Genetic algorithm, Fuzzy-Genetic which is a combination of Fuzzy logic and Genetic algorithm, are widely used in modeling intelligent systems for medical diagnosis and prognosis.

\section{Neuroscience and Neurocomputing}

Neurocomputing also known as artificial neural network or connectionist is a mathematical representation of the human neural architecture, reflecting its "learning" and "generalization" abilities. For this reason, ANNs belong to the area of computational intelligence/artificial intelligences. It also belong to a machine learning algorithms. Neural network are widely applied in model highly non-linear systems in which the relationship among the variables is unknown or very complex [1].

Neuroscience also known as the science of brain. Human brain is a source of natural intelligence and a truly remarkable parallel computational system. Brain cells function about $10^{6}$ times slower than electronic circuit gates, but human brains process visual, sense of touching and auditory information much faster than any modern computer 
could do in millions of years. The method behind this speed is the number of the neurons which is around $10^{10}$ with the number of connections. As shown in figure 1.
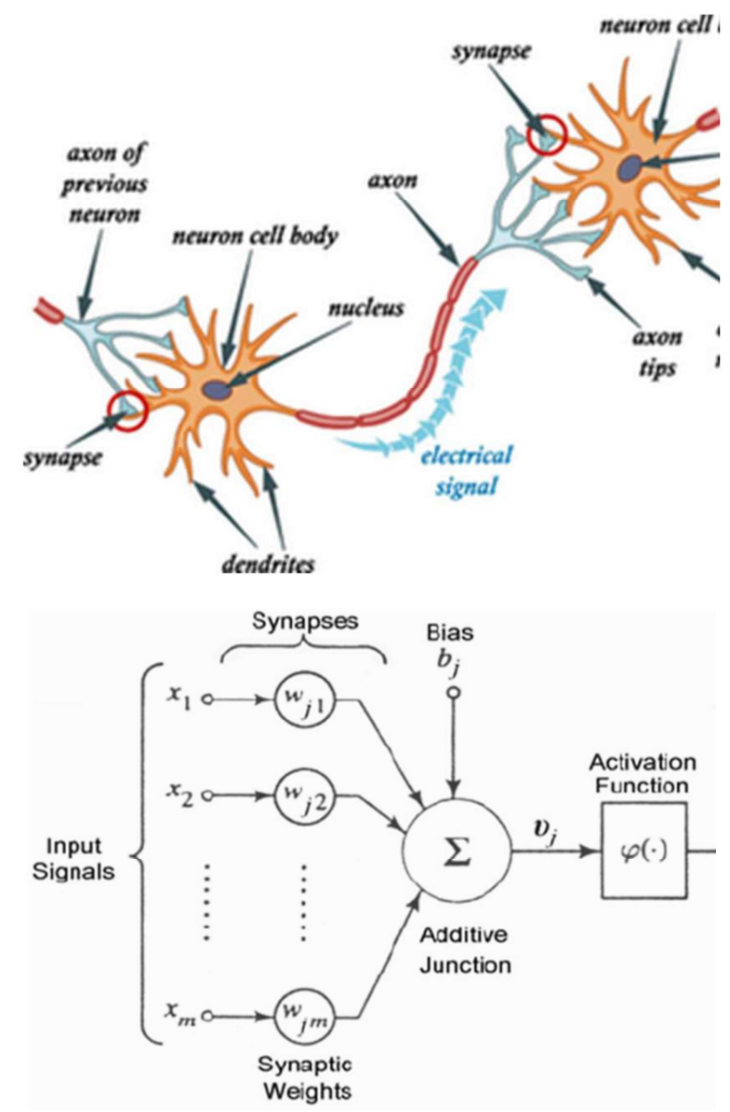

Figure 1. Biological neurons and artificial neural network [36].

Most of the computational intelligence techniques that are applied to medical diagnosis consider symptoms, biochemical analysis in labs and other feature like age, gender, as an inputs parameters to the systems, these would be further define with some linguistic variables like mild, moderate, severe, very severe, old, young, and so on, numeric values are assign to this variables which would form a datasets for training and testing of the system as illustrated in figure 1 below; where Artificial Neural Network are applied to medical diagnosis.

\section{INPUT}

\section{OUTPUT}

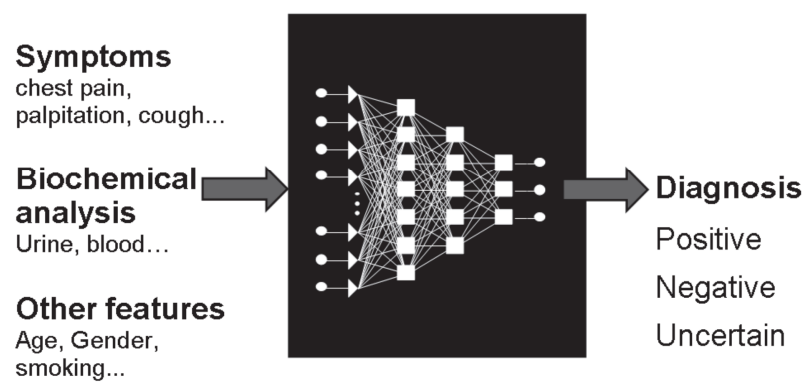

Figure 2. Detail of inputs and output parameters in ANN-based diagnosing system [1].

Artificial Neural Network is machine learning algorithms that require datasets for training and testing. It is made up of inputs layers, hidden layers and the output layers. In inputs layers every input has some weight which are subject to adjustment.

\section{Fuzzy Logic}

The mathematical foundation of fuzzy logic rest on the shoulder of fuzzy sets. Fuzzy set was first introduced by Lofti Zadeh in which it derived from classical set theory. Fuzzy logic consist of inputs parameters knowledge based and outputs the number of inputs and output are strongly depend on the complexity of the system build. The inputs parameter has some linguistic variables which would be used to build the rules for the system. In fuzzy logic membership functions are also required such as trapezoidal, Gaussian, Singleton, Triangular etc. Fuzzy logic are also applied in medical diagnosis using either of its two methodologies that is Sugeno and Mamdani. The figure 2 below illustrates how Sugeno model used in medical diagnosis;

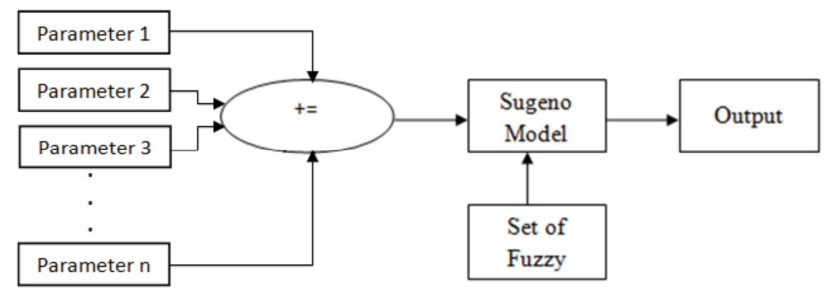

Figure 3. Fuzzy Sugeno model for disease diagnosis [48].

\subsection{Membership Functions}

In fuzzy logic there many membership functions applied to different research area such as Gaussian, triangular, trapezoidal, singleton among others which are all presented mathematically and graphically.

\subsection{Triangular Membership Function}

Triangular is a linear membership function illustrated in the figure below; where $\mathrm{a}, \mathrm{b}$ and $\mathrm{c}$ represent the $\mathrm{x}$ coordinates of the three vertices of $\mu \mathrm{A}(\mathrm{x})$ in the fuzzy set $\mathrm{A}$. The coordinate " $\mathrm{a}$ " is defined as the lower boundary in set A whose degree of membership is zero. The coordinate " $c$ " is defined as the upper boundary whose degree of membership is also zero. Finally, coordinate " $b$ " is the third apex of the triangle whose degree of membership is one. [66] As shown in the figure 4 below;

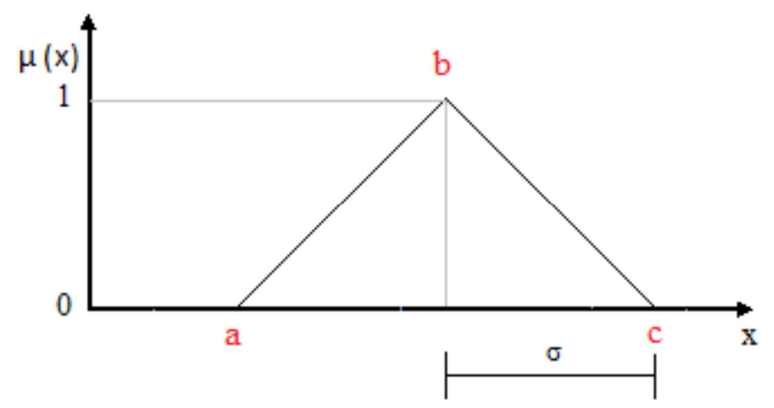

Figure 4. Triangular membership function. 
Mathematically can be represented as;

$$
f(x ; a, b, c)=\max \left\{\min \left(\frac{x-a}{b-a}, \frac{c-x}{c-b}\right), 0\right\}
$$

\subsection{Gaussian Membership Function}

The Figure 5 below; illustrates a typical Gaussian membership function where $\mathrm{x}$ is the input variable, $\mathrm{b}$ is the center of the membership function and $\sigma$ is the constant that represents the width of the membership function [66].

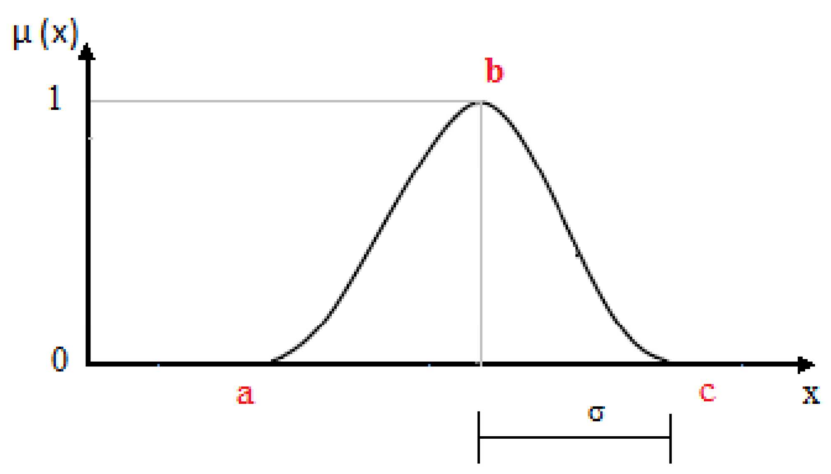

Figure 5. Gaussian membership function.

Mathematically can be represented as

$$
f(x ; \sigma, c)=e^{\frac{-(x-c)^{2}}{2 \sigma^{2}}}
$$

\subsection{Bell-Shaped Membership Function [67]}

A bell-shaped MF has symmetric shape and is given by the expression

$$
f(x ; a, b, c)=\frac{1}{1+\left|\frac{x-c}{a}\right|^{2 b}}
$$

\subsection{A Trapezoidal Membership Function [67]}

A trapezoidal MF is described by four parameters; $a, b, c$, and $d$ and it is given by the expression

$$
f(x, a, b, c, d)=\max \left\{\min \left(\frac{x-a}{b-a}, 1, \frac{d-x}{d-c}\right), 0\right\}
$$

\section{Soft Computing}

In soft computing that is when two computational intelligence techniques are applied to medical diagnosis for example Neuro-fuzzy are applied in diagnosis of tuberculosis as shown in figure 3 below;

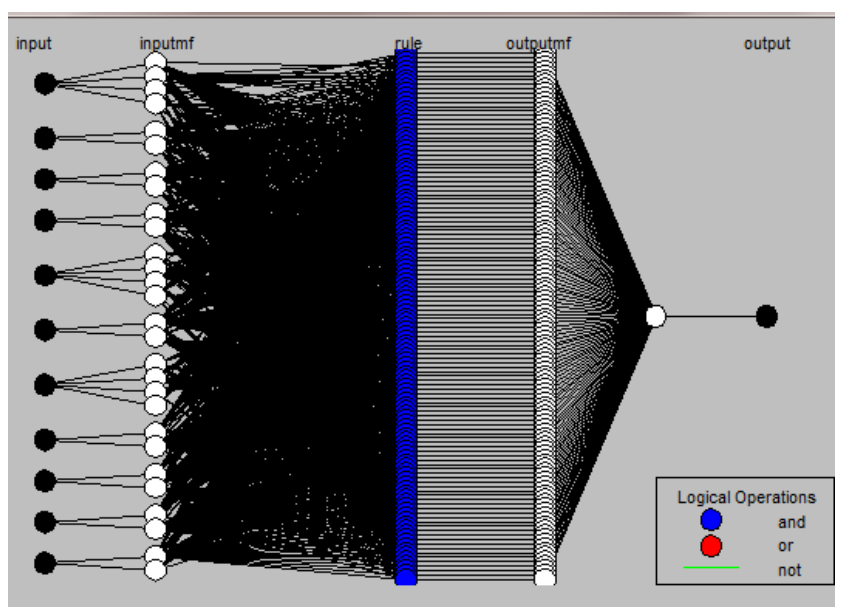

Figure 6. Neuro-fuzzy models for TB diagnosis [27].

\section{Machine Learning Algorithms Applied to Medical Diagnosis}

In [38] Developed knowledge-based Expert system for diagnosing tropical infectious diseases on web based platform to receive input in the form of physical symptoms and results of complete blood examination in the laboratory. [16] Developed a Fuzzy Inference System model for medical diagnosis in the area of psychology and psychiatry. [17] Presented a kidney diseases Diagnosis system using fuzzy logic the results of the research indicated that, in the diagnosis of eight cases of kidney diseases through the examination of 21 indicators using fuzzy logic. [9] Developed a decision support system which predicts the possibility of heart disease risk of patients for the next tenyears using fuzzy logic and decision tree. [41] Used artificial neural networks with six factors (smoking, age, weight, height, sex and High blood lipid) for high blood pressure. [2] Developed an adaptive Neuro-Fuzzy Inference System for Diagnosis of Ebola Hemorrhagic Fever.

In the work of [3] developed a medical diagnostic system using Visual Prolog Programming language. [54] Fuzzy Abdominal Obesity models were developed on the basis of Body Mass Index, Waist Circumference and Weight to Height ratio measurements. [5] Developed a Fuzzy Expert system with 8 fuzzy rules for the diagnosis of hypertension. Fuzzy logic was applied in the work of [64] to health monitoring system using wireless sensor network for deciding final observation of patient health status based on bio-signal reading. [65] Have determined the relationship between risk factors (insulin, leptin, homocysteine, and urinary albumin excretion) and circadian Blood Pressure variations in essential hypertensive subjects. In [47] Artificial Immune Recognition System (AIRS) with fuzzy labeling was used to form an unsupervised learning AIRS approach and to substantiate tuberculosis diagnosis identification by using clinical tuberculosis diagnostic data sets. [15] Developed an expert system for human disease diagnosis it works on the patient data by combining production rules and a neural network. [19] investigated the risk factors for the 
development of diabetes mellitus, the hypertension, and the dyslipidemia simultaneously in a community-based observational cohort study using sex, age, Body Mass Index, smoking, alcohol and exercise parameters. [59] Proposed a Hybrid system for diagnosis chest diseases using Artificial Neural Network (ANN) and Genetic Algorithm (GA). In the research of [61] they have developed a Web based Fuzzy inference tool that evaluates the risk of exacerbating cardiovascular disease.

In [4] Develop an expert system for diagnosis of diseases thyroid cancer by combining the methods of neural network and certainty factor, In the research of [18] they have developed expert systems for diagnosis of asthma disease by the method of Fuzzy Logic. [32] Developed expert system for diagnosis of heart disease by the method of Fuzzy Logic. [60] In his work titled "Fuzzy Expert System for Medical Diagnosis" [14] developed a web-based Fuzzy Expert System for the public to diagnose the hypertension risk. [63] tested a logistic regression model to examine the independent influence of changes in body mass index, health related behaviors and social risk factors on changes in self-reported diagnosis of hypertension with using body mass index, physical activity, smoking, alcohol, acute condition, memory status, gender, residence, education, marital status, and income parameters.

\section{Systematic Literature Review}

Machine learning algorithms or Computational intelligence techniques are recently applied for classification, prediction of the presence of microorganism, biomedical medical image extraction, selection, and Medical diagnosis, optimization of medical system, estimation, pattern recognition, testing, data mining, and determination of severity level of disease among others. The contributions of authors that applied computational intelligence and/or Artificial intelligence techniques and soft computing to medical diagnosis are systematically summarize in the table 1 below;

Table 1. Summary of Literature review.

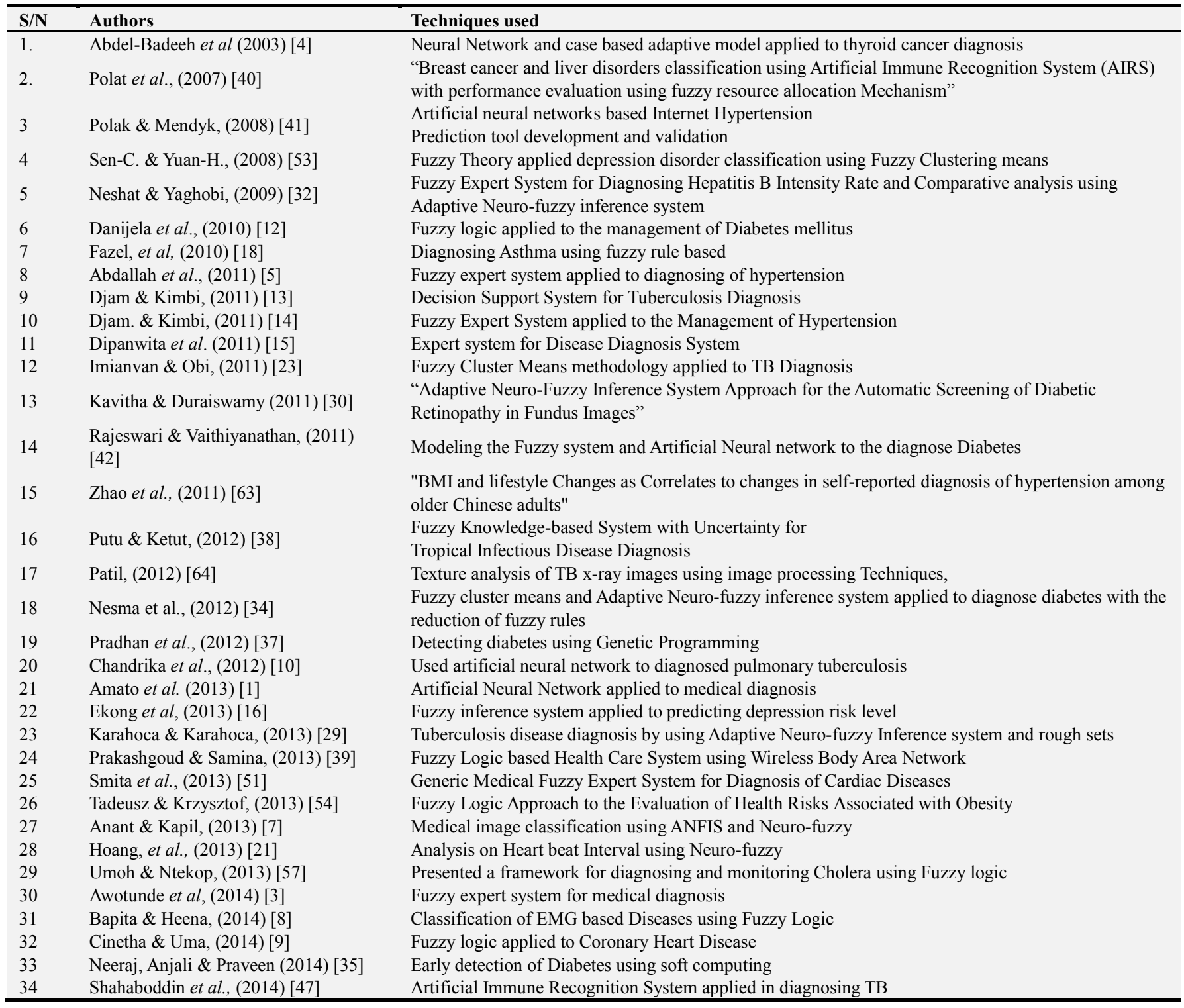




\begin{tabular}{|c|c|c|}
\hline $\mathbf{S} / \mathbf{N}$ & Authors & Techniques used \\
\hline 35 & Annie et al, (2015) [2] & Diagnosis of Ebola using Adaptive Neuro-fuzzy inference system \\
\hline 36 & Adlassnig, (2015) [6] & Medical diagnosis using fuzzy sets theory \\
\hline 37 & Filipe \& Henrique, (2015) [20] & Artificial Neural network applied to diabetes control \\
\hline 338 & Mahmoud et al, (2015) [31] & Artificial Immune recognition system applied to TB identification \\
\hline 39 & Farzad, (2015) [17] & Fuzzy logic applied to kidney disease diagnosis \\
\hline 40 & Navneet et al, (2015) [33] & Fuzzy Based Decision Support System applied in identification of TB \\
\hline 41 & Vally \& Sarma, (2015) [59] & Neuro-genetic technique applied in diagnosing chest disease \\
\hline 42 & Varinder, (2015) [60] & Medical Diagnosis using fuzzy system \\
\hline 43 & Vaishali \& Supriya (2015) [58] & Diagnosing diabetes using Fuzzy logic \\
\hline 45 & Tejashri \& Satish (2015) [58] & Neural Network, fuzzy logic and Gaussian Kernel methodologies applied to the prognosis of diabetes \\
\hline 46 & Yuanda et al., (2015) [61] & Risk Assessment of Cardiovascular Disease using web-based fuzzy system \\
\hline 47 & Zeinab \& Hamid, (2015) [62] & Fuzzy and Neural network used in diagnosing of Hypertension \\
\hline 48 & Chanchal \& Tejalal, (2015) [11] & Implement fuzzy web based system for diagnosing Epistaxis \\
\hline 49 & Ricard et al., (2015) [46] & Malaria Diagnosis using ANFIS \\
\hline 50 & Iryna P. et al. (2016) [25] & Neuro-fuzzy techniques used medical diagnosis task with online mode \\
\hline 51 & Kamble et al, (2016) [28] & Image Processing technique used in detecting TB \\
\hline 52 & Ramana \& Prasad, (2016) [45] & Radial Basis and fuzzy logic used for detection and diagnosis of lung cancer \\
\hline 53 & Rupali \& Jyoti, (2016) [49] & Soft computing applied in predicting TB \\
\hline 54 & Radek et al. (2016) [50] & Adaptive Neuro-fuzzy inference system used in extraction of fetal electrocardiogram \\
\hline 55 & Tran et al., (2016) [56] & Clustering combined with fuzzy ruled based applied to medical diagnosis from dental X-ray \\
\hline 56 & Shashank et al., (2016) [48] & Neuro-fuzzy applied to e-health for monitoring disease diagnosis \\
\hline 57 & Ibrahim et al.. (2017) [24] & Neuro-fuzzy model used to predict the presence of Mycobacterium TB \\
\hline 58 & Jerome et al., (2017) [26] & Adaptive Neuro-fuzzy used in determine the severity level of osteomyelitis \\
\hline 59 & Jerome et al., (2018) [27] & Neuro-fuzzy methodology applied to TB diagnosis and control \\
\hline
\end{tabular}

\section{Wireless Sensor Network}

The recent development of high performance microprocessors and novel sensing materials has stimulated great interest in the development of smart sensors physical, chemical, or biological sensors combined with integrated circuits. It is not uncommon to place multiple sensors on a single chip, with the integrated circuitry of the chip controlling all these sensors [68]. An emerging application for wireless sensor networks involves their use in medical care. In a hospital or clinic, outfitting every patient with tiny, wearable wireless vital sensors would allow doctors, nurses and other caregivers to continuously monitor the status of their patients. In an emergency or disaster scenario, the same technology would enable medics to more effectively care for large numbers of casualties. First responders could receive immediate notifications on any changes in patient status, such as respiratory failure or cardiac arrest. Wireless sensors could replace existing wired telemetry systems for many specific clinical applications, such as physical rehabilitation or long-term ambulatory monitoring [69].

\subsection{Types of Wireless Sensor Network}

[70] itemize four (4) types of Wireless Sensor Network as follow;

i. Terrestrial Wireless Sensor Network

ii. Underground Wireless Sensor Network

iii.Multi-media Wireless Sensor Network

iv. Mobile Wireless Sensor Network.

\subsubsection{Terrestrial Wireless Sensor Network}

This type of sensor is mainly applicable in the environmental monitoring and surveillance [71].

\subsubsection{Underground Wireless Sensor Network}

This type of sensor is usually applicable in agricultural practices [71].

\subsubsection{Underwater Wireless Sensor Network}

This type of network are mostly deployed in hostile environment and it suffered a lot of communication problems due to the nature of the environment [71].

\subsubsection{Multimedia Wireless Sensor Network}

This type of sensor are applicable to computer graphics which includes images, videos and audios [71].

\subsubsection{Mobile Wireless Sensor Network}

This type of sensor has the ability to move from one point to another [71].

\subsection{Architecture of Wireless Sensor Network}

Basically we have two types of architecture used in wireless sensor network this are flat and hierarchical architecture

\subsubsection{Flat Architecture}

In flat architecture, except for sink node, the other nodes are identical, they have the same capacity in terms of energy and computing, also, they have the same role in sensing task. Node can directly communicate with a sink in single hop manner or communication with sink can be in multi hops manner [71].

\subsubsection{Hierarchical Architecture}

A hierarchical architecture to deploy a large number of sensors. The network is divided into several groups or clusters which are the organizational unit of the network. Depending of cases, a more expensive cluster node type and more powerful than other nodes or a normal node in the 
cluster is designated as group leader called cluster head that is responsible of coordination of the sensors under its responsibility and act as a gateway to another cluster [71].

\section{Application of Wireless Sensor Network to Health Care Systems}

In the research of [72] Improving Health Care through Mobile Medical Devices and Sensors recommends that Mobile technology offers interesting ways to help with health care access, affordability and service delivery. [73] In their research paper they have identified challenges those developing countries particularly Tanzania will face while adopting e-healthcare. [73] agree that apart from the identified challenges; privacy, security and confidentiality are among the common challenges to both developed and developing countries. [74] They conduct a surveyed on the existing middleware design approaches for wireless sensor networks. [75] In their research paper present a monitoring system that has the capability to monitor physiological parameters from multiple patient bodies. [76] Developed a robust, scalable software platform for medical sensor networks, called Code Blue. [77] Believed that with all new technologies we face with problem of failure or success. Despite the promising features, risks of failure are always present. [78] In their paper, medical sensors were used to collect physiological data from patients and transmit it to Intelligent Personal digital Assistant (IPDA) using ZigBee/IEEE802.15.4 standard and to medical server using $3 \mathrm{G}$ communications. [79] Believed that the application of the Wireless Sensor Networks in healthcare systems is divided into three categories: monitoring of patients in clinical settings, home and elderly care center monitoring for chronic and elderly patients, and collection of long-term databases of clinical data. [80] In their research paper; they describe the potential of biomedical smart sensors they also explain the challenges for wireless networking of human embedded smart sensor arrays and the preliminary approach for wireless networking of retina prosthesis. [81] Their research paper evaluate the integration of Cloud computing, wireless sensor technology and Internet of Things in a healthcare environment.

\section{Conclusion}

Machine learning algorithms or Computational intelligence techniques are recently applied for classification, prediction of the presence of microorganism, biomedical medical image extraction, selection, and Medical diagnosis, optimization of medical system, estimation, pattern recognition, testing, data mining, and determination of severity level of disease among others. The contributions of authors that applied computational intelligence and/or Artificial intelligence techniques and soft computing to medical diagnosis are systematically. However, in this survey a system review were also conducted on application of wireless sensor network in health care services then we proposed that combining machine learning algorithms and wireless sensor network would improve the performance of health care system.

\section{References}

[1] F. Amato, Alberto L., Eladia M., Petr V., Ales H. and Josef H., Artificial Neural Networks in Medical Diagnosis Journal of Applied Biomedicine 11, (2013) 47-58.

[2] O. Annie E. and Jonathan C. O., "An Adaptive Neuro-Fuzzy Inference System for Diagnosis of Ebola Haemorrhagic Fever" The Pacific Journal of Science and Technology 16 (1), 251-261, 2015.

[3] J. Awotunde. B., Matiluko O. E., Fatai O. W, "Medical Diagnosis System using Fuzzy Logic African Journal of Computing and ICT, 7 (2), 99-106. 2014.

[4] M. Abdel-Badeeh Salem, Bassant M. El. Bagoury, "A CaseBased Adaption Model for Thyroid Cancer Diagnosis Using Neural Networks", FLAIRS, 155-159. 2003.

[5] A. Abdullah. A. A, Zakaria. Z, Mohammad. N. F., "Design and Development of Fuzzy Expert System for Diagnosis of Hypertension".. IEEE Xplore. Intelligent Systems, Modeling and Simulation, 113-117. 2011.

[6] K. Adlassnig K., "Fuzzy Set Theory in Medical Diagnosis, " IEEE Transactions on Systems, Man, and Cybernetics, (1986), pp. 260-265. International Journal of Bio-Science and BioTechnology, 7 (6), 2015.

[7] B. Anant, Kapil K. S., "An Approach to Medical Image Classification Using Neuro Fuzzy Logic and ANFIS Classifier", International Journal of Computer Trends and Technology, 4 (3), 236-240, 2013.

[8] P. Bapita P, Heena, "Classification of EMG based Diseases using Fuzzy Logic at Second Level" International Journal of Computer Applications, 91 (3) (2014) 25-33.

[9] K. Cinetha, Uma P. M, "Decision Support System for Precluding Coronary Heart Disease (CHD) Using Fuzzy Logic" International Journal of Computer Science Trends and Technology, 2 (2), 102-107. 2014.

[10] V. Chandrika V., Parvathi C. S. and P. Bhaskar, "Diagnosis of Tuberculosis Using MATLAB Based Artificial Neural Network" IJIPA: 3 (1), 37-42, 2012.

[11] S. Chanchal S., Tejalal C., "A Web Based Fuzzy Expert System for Epistaxis Diagnosis" International Journal of Computer Science and Information Technologies, 6 (4), 40624068, 2015.

[12] T. Danijela, Pedrag P., Aleksandar D., “A Fuzzy Approach to Evaluation and Management of Therapeutic Procedure in Diabetes Mellitus Treatment" Yugoslav Journal of Operations Research, 20 (1), 99-116, 2010.

[13] X. Djam Y, Kimbi Y. H., “A Decision Support System for Tuberculosis Diagnosis". The Pacific Journal of Science and Technology, 12 (2), 410-425. 2011.

[14] X. Djam Y., Kimbi Y. H., "Fuzzy Expert System for the Management of Hypertension", The Pacific Journal of Science and Technology, 12 (1), 390-402. 2011. 
[15] B. Dipanwita et al., "Disease Diagnosis System", International Journal of Computer Science \& Informatics, 1 (2), (2011) 2430. 2011.

[16] V. Ekong E., Uyinomen O., Uwadiae, Enobakhare E., Abasiubong, Festus, Onibere, Emmanuel A., "A Fuzzy Inference system for predicting depression risk levels" African Journal of mathematics and computer Science Research, 6 (10) (2013) 197-204. 2013.

[17] F. Farzad J., "Kidney Diseases Diagnosis by Using Fuzzy Logic" Proceedings of the 2015 International Conference on Industrial Engineering and Operations Management Dubai, United Arab Emirates (UAE), March (2015) 3- 5, 2015.

[18] M. Fazel H. Z., Zolnoori M., Moin M., Heidarnejad H., "A Fuzzy Rule-Based Expert System for Diagnosing Asthma", Transaction E. Industrial Engineering, 17 (2010) 129-142. 2010 .

[19] M. Fukui, Tanaka. M, Toda. H, Senmaru. T, Sakabe. K, Ushigome. E, Asano. M, Yamazaki. M, Hasegawa. G, Imai. S, Nakamura. N.,. "Risk factors for development of diabetes mellitus, hypertension and dyslipidemia“, Diabetes Research and Clinicl Practice, 94 (1), 2011.

[20] F. Filipe, Henrique Vecente, "Artificial Neural Networks in Diabetes" Control Science and Information Conference (2015) July 28-30, 2015 London, UK, 2015.

[21] C. Hoang, Thuan N., Trung L., "Neuro-Fuzzy Approach to Heart Rate Variability Analysis" International Journal of Bioscience, Biochemistry and Bioinformatics, 3 (5) (2013) 456-459.

[22] G. Irene S., Mark L. W., "Current Concepts in the Management of Tuberculosis" Mayo Clinical Proceeding, 86 (4), 348-361, 2011.

[23] A. Imianvan. A, Obi J. C., "Fuzzy Cluster Means Expert System for the Diagnosis of Tuberculosis". Global Journal of Computer Science \& Technology, 11 (6), 41-48. 2011.

[24] G. Ibrahim, Jerome M. G., Timothy U. M. "Designing a Neuro-Fuzzy Model For Predicting the Existence of Mycobacterium Tuberculosis" $36^{\text {th }}$ Annual Conference of the Nigerian Mathematical Society (NMS) $9^{\text {th }}-12^{\text {th }}$ May (2017) held at University of Agriculture Makurdi, Benue State. 2017.

[25] P. Iryna et al., "Neo-Fuzzy Approach for Medical Diagnostics Tasks in Online-Mode" IEEE First International Conference on Data Stream Mining \& Processing 23-27 August (2016) Lviv, Ukraine, 34-37. 2016.

[26] M. Jerome G, Ibrahim Goni, Timothy U. M., "Adaptive Neuro-fuzzy system for determining the severity level of osteomyelitis and control", Archives of Applied Science Research, 9 (2), 9-15, 2017.

[27] M. Jerome G., Ibrahim G., Mohammed I. "Neuro-Fuzzy Approach for Diagnosing and Control of Tuberculosis" the International Journal of Computational Science, Information Technology and Control Engineering (IJCSITCE) 5 (1) 2018.

[28] P. Kamble A., Anagire V. V., Chamtagoudar N. S. "CXR Tuberculosis Detection Using MATLAB Image Processing" International Research Journal of Engineering and Technology (IRJET) 3 (6) (2016) 2342-2344.

[29] A. Karahoca, Karahoca D, "Tuberculosis disease diagnosis by using Adaptive Neuro- Fuzzy Inference System and Rough Sets" 471-483. 2013.
[30] S. Kavitha S., Duraiswamy k., "Adaptive Neuro-Fuzzy Inference System Approach for the Automatic Screening of Diabetic Retinopathy in Fundus Images", Journal of Computer Science, 7 (7), 1020-1026, 2011.

[31] R. Mahmoud S., Shahaboddin S., Shahram G., Teh Y., Aghabozorgi S., Laiha M., Valentina E., "RAIRS2 A new Expert System for Diagnosing Tuberculosis with Real world Tournament Selection Mechanism inside Artificial Immune recognition system" International Federation for Medical and Biological Engineering Springer 2015.

[32] M. Neshat, Yaghobi M., "Designing a Fuzzy Expert System of Diagnosing the Hepatitis B Intensity Rate and Comparing it with Adaptive Neural Network Fuzzy System", in World Congress on Engineering and Computer Science (WCECS), Vol. II. 2009.

[33] W. Navneet, Sharad K. T. and Rahul M., "Design and Identification of Tuberculosis using Fuzzy Based Decision Support System" Advances in Computer Science and Information Technology, 2 (8), 57-62, 2015.

[34] S. Nesma, Meryem S., and Mohamed A. C, "Interpretable Classifier of Diabetes Disease" International Journal of Computer Theory and Engineering, 4 (3) 438-442, 2012.

[35] K. Neeraj G., Anjali G., Praveen K. T. "Early Detection of Diabetes Patients using Soft Computing IEEE International Conference on Issues and Challenges in Intelligent Computing Techniques, 2014.

[36] D. Nikolaos M., "Adaptive neuro-fuzzy inference systems (ANFIS) applied on medical diagnosis". B. Sc. Electrical and Computer Engineer Project, Technical University of Crete (2017).

[37] Pradhan et al., "A Genetic Programming Approach for Detection of Diabetes International" Journal of Computational Engineering Research, 2 (6), 91-94 2012.

[38] M. Putu P., Ketut D. P. "Fuzzy Knowledge-based System with Uncertainty for Tropical Infectious Disease Diagnosis" International Journal of Computer Science 9 (3), (2012) 157163.

[39] P. Prakashgoud, Samina M., "Fuzzy Logic based Health Care System using Wireless Body Area Network" International Journal of Computer Applications 80 (13), 46-51. 2013.

[40] K. Polat et al., "Breast Cancer and Liver Disorders Classification using Artificial Immune Recognition System (AIRS) with Performance Evaluation by Fuzzy Resource Allocation Mechanism" Expert System Application 32, 172183. 2007.

[41] S. Polak., and Mendyk. A., Artificial Neural Networks Based Internet Hypertension Prediction tool development and validation, Applied Soft Computing 8 (1), 734-739. 2008.

[42] K. Rajeswari, Vaithiyanathan V., "Fuzzy based Modeling for Diabetic Diagnostic Decision Support using Artificial Neural Network" International Journal of Computer Science and Network Security, 11 (4), 126-130, 2011.

[43] M. Ramana N. V. \& Prasad B. M. S. "A Novel Hybrid Application of Radial Basis Function Neural Network and Fuzzy Logic for Detection and Diagnosis of Lung Cancer" International Journal of Engineering Science and Computing. (2016) 2222-2224, 2016. 
[44] A. Richard A., Joseph K. P. \& Kwabena R., "Implementation of Adaptive Neuro Fuzzy Inference System for Malaria Diagnosis (Case Study: Kwesimintsim Polyclinic)", International Journal of Computer Applications, 115 (7), 3337, 2015.

[45] S. Shahaboddin. Somayeh H., Hossein J., Mohsen A., Shaghayegh V., Dalibor P., Abdullah G. \& Laiha M. Tuberculosis Disease Diagnosis Using Artificial Immune Recognition System International Journal of Medical Sciences, 11 (5), 508-514, 2014.

[46] B. Shashank B., Praveen K. \& Amit K. G., "Neuro-fuzzy expert system in e-Health Monitoring for Disease Diagnosis" International Journal of Innovations in Engineering and Technology, 7 (2), 249-252, 2016.

[47] Z. Rupali, Jyoti A., "Pre-Prediction of Tuberculosis Disease Using Soft Computing Technique" International Journal of Advanced Research in Computer and Communication Engineering. 5 (6), 56-61, 2016.

[48] M. Radek et al., "Fetal ECG Extraction Based on Adaptive Neuro-Fuzzy Interference System" IEEE $10^{\text {th }}$ International Symposium on Communication Systems, Networks and Digital Signal Processing 2016.

[49] S. Smita S. S., Sushil S. \& Ali M. S., "Generic Medical Fuzzy Expert System for Diagnosis of Cardiac Diseases" International Journal of Computer Application 66 (13) 35-44. 2013.

[50] S. Shahaboddin S. Somayeh H., Hossein J., Mohsen A., Shaghayegh V., Dalibor P., Abdullah G. \& Laiha M., "Tuberculosis Disease Diagnosis Using Artificial Immune Recognition System" International Journal of Medical Sciences 11 (5), 508-514 2014.

[51] C. Sen-C., Yuan-H. L., Applications of Fuzzy Theory on Health Care: An Example of Depression Disorder Classification Based on FCM WSEAS Transactions on Information Science \& Applications, 5 (1), 31-36. 2008.

[52] Tadeusz N. and Krzysztof P., "A Fuzzy Logic Approach to the Evaluation of Health Risks Associated with Obesity" Proceedings of the 2013 Federated Conference on Computer Science and Information Systems, 231-234. 2013.

[53] N. Tejashri N. G. \& Satish R. T., Prognosis of Diabetes using Neural Network, Fuzzy Logic, Gaussian Kernel Method International Journal of Computer Applications 124 (10), 33$36,2015$.

[54] M. Tran T. et al., "Medical Diagnosis from Dental X-Ray Images: A Novel Approach Using Clustering Combined with Fuzzy Rule-based Systems” IEEE 2016.

[55] U. Umoh U. A. \& Ntekop M. M., "A Proposed Fuzzy Framework for Cholera Diagnosis and Monitoring", International Journal of Computer Applications 82 (17) 1-10, 2013.

[56] J. Vaishali J. \& Supriya R., Improving the Prediction Rate of Diabetes using Fuzzy Expert System International Journal of Information Technology and Computer Science, 10 (2015) 8491, 2015.

[57] D. Vally. and CH V Sarma, "Diagnosis Chest Diseases Using Neural Network and Genetic Hybrid Algorithm" International Journal of Engineering Research and Applications,. 5 (1) 20 26. 2015 .
[58] P. Varinder, "Fuzzy Expert System for Medical Diagnosis "International Journal of Scientific and Research Publications, Vol. 5 (1) 2250-315, 2015.

[59] L. Yuanda, David P. and Jorge M., "A Web-based Fuzzy Inference System Based Tool for Cardiovascular Disease Risk Assessment" NOVA. 13 (24) 7-16. 2015.

[60] A. Zeinab A. and Hamid T., "Design of a Fuzzy Expert System and a Multi-Layer Neural Network System For Diagnosis of Hypertension Bulletin of Environment", Pharmacology and Life Sciences 4 (15) 138-145 2015.

[61] A. Zhao S. N., H., Smith. M. L, Ory. M. G., Phillips. C. D., "BMI and Lifestyle Changes as Correlates to Changes in Selfreported Diagnosis of Hypertension among older Chinese Adults", Journal of the American Society of Hypertension. 5 (1) 21-30. 2011.

[62] S. Patil. A., "Texture Analysis of TB x-ray Images using Image Processing Techniques", Journal of Biomedical and Bioengineering, 3 (1), 53-56. 2002.

[63] C. Tsioufis, Antoniadi. D, Stefanadis C, Tzioumis K, Pitsavos C, Kallikazaros I, Psarros. T, Lalos S, Michaelides A. \& Toutouzas P., "Relationships Between New Risk Factors and Circadian Blood Pressure Variation in Untreated Subjects With Essential Hypertension" American Journal of Hypertension, 15 (7), 600-604. 2002.

[64] F. Marcus F. "The Application of Fuzzy Logic in Determining Linguistic Rules and Associative Membership Functions for the Control of a Manufacturing Process", M. Engr. Dissertation Dublin Institute of Technology India. 2011.

[65] Z. Jin, \& Bimal, K. B. "Evaluation of membership functions for fuzzy logic controlled induction motor drive". IEEE Spectrum. 2 (1), 229-234 2012.

[66] S. Loren, Sandeep K. S. G., \& Jennifer W. "Research challenges in wireless Networks of Biomedical sensors", Rome Italy. 2001.

[67] S. Victor, Bor-rong C., Konrad L., Thaddeus R. F. F. and Matt W. "Sensor Networks for Medical Care Technical" Report TR-08-05, Division of Engineering and Applied Sciences, Harvard University. 2005.

[68] S. Neelam "Challenges of next generation wireless sensor networks and its impacts on society" Journal of Telecommunications, 1 (1). 2010.

[69] A. Ado A., Abdelhak G., Nabila L. and Blaise O. Y. "Concepts and Evaluation of Research in the field of Wireless Sensor Network" International Journal of Computer Networks \& Communication (IJCNC) 7 (1) p. 81-98. 2015.

[70] M. Darrel "Improving Health care through Mobile Medical Devices and Sensor. Center for Technology innovation at Brookings" p. 1-13. 2013.

[71] O. Zanifa, Dennis L., Fredrick M., and Bing W. "Analysis of the Challenges affecting E-Healthcare Adoption in Developing Countries; A case of Tanzania" International Journal of Information Studies Vol. 2. 38 -50, 2010.

[72] B. Bhaskar B., Hiren K. D. S., Nityananda S. "A Survey on Middleware for Wireless Sensor Network" Journal of Wireless Networking and Communications, 4 (1): 7-17. 2014. 
[73] M. Aminian, Naji H. R "A Hospital Healthcare Monitoring System Using Wireless Sensor Networks". Journal of Health Med Inform 4: 121. 2013.

[74] W. Matt, "A Wireless Sensor Network Infrastructure for Medical Application". Division of engineering and Applied Science Harvard University. 2015.

[75] S. Stanković, "Medical Applications Based on Wireless Sensor Networks" pp. 1-5, 2014.

[76] P. Ademola A., Nureni A. A., Ademola O., A., Kehinde K. A., Henry O. N. "Using Wearable Sensors for Remote Healthcare Monitoring System". Scientific Research Journal of Sensor Technology, pp. 22-28. 2011.
[77] M. Afsaneh, Ali S. M., Paymon S. M., \& Reza S., "Application of Wireless Sensor Network in Healthcare system" 120th ASEE Annual Conference \& Exposition, 23-26, 2013.

[78] S. Loren, Sandeep K. S. G., Jennifer W. "Research challenges in wireless Networks of Biomedical sensors" ACM SIGMOBILE 7/01 Rome Italy. 2001.

[79] A. Nawaf, Anthony S. A., Justin C. "Use of Cloud Computing with Wireless Sensor Networks in an Internet of Things Environment for a Smart Hospital Network". The Seventh International Conference on eHealth, Telemedicine, and Social Medicine pp. 52-58. 2015. 